\title{
Epidemiology of vaginal prolapse in mixed age ewes in New Zealand
}

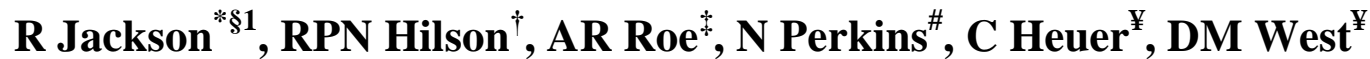

\author{
* 5 Kensington Mews, Palmerston North 4410, New Zealand \\ $\dagger$ Vet Services (Hawkes Bay) Ltd, PO Box 503, Waipukurau 4200, New Zealand \\ $\ddagger$ Vet South Ltd, Winton, New Zealand \\ \# Aus Vet Animal Health Services Pty Ltd, PO Box 1278, Toowoomba, Queensland, 4350, Australia \\ ${ }^{¥}$ Massey University Institute of Veterinary Animal \& Biomedical Sciences, Private Bag 11222 , \\ Palmerston North, New Zealand \\ ${ }^{\S}$ Author for correspondence. Email: Ron.Jackson@xtra.co.nz
}

\section{Supplementary Information}

\section{Classification of breeds.}

At the time of the study many farmers were transitioning from traditional breeds and predominately Romney flocks to other breeds. Some breeds viz. Drysdale ewes, Composite ewes, Drysdale-cross ewes, Drysdale and Hampshire rams and Drysdale-cross and Hampshire-cross rams were disregarded due to low frequencies of occurrence. Flocks were further classified as dual, terminal or mixtures of dual and terminal on the basis of the types of rams used. For example, a Romney flock using Romney or Perendale or Finn rams was classified as dual purpose, a Perendale flock using Perendale as well as Downs, Texel or East Friesian rams was classified as a mixture of dual and terminal while a Romney flock using terminal sires was classified as a terminal type flock.

\section{TABLE 5: effect of other variables.}

The adjusted incidence measures and CIs are derived from inverse transformation of the LSM estimates derived from the multivariable statistical model. It can be seen in Table 5 that the predicted mean incidence estimates of annual incidence are different to the crude mean estimates. This is because they take into account the effect of other variables in the model and the random effect of farm.

For example, simply summing the number of cases over all farms in year 2000 and dividing that number by the total number of sheep on all farms in that year produces a crude incidence estimate of 1.21 cases per 100 ewes $(2,278 / 188,169)$. But this estimate does not take into account the potential effect of other factors such as region and terrain. The adjusted incidence estimate for the same period is 0.57 cases per 100 ewes. This estimate is considered more likely to represent an unbiased measure of the effect of year 2000 since it takes into account the effect of other risk factors. Similarly the model predicts that flocks that are shorn within 90 days before the start of mating will experience 0.14 fewer cases per 100 ewes than flocks that are not shorn in that period.

\section{Interpretation of body weights:}

Weights in ruminants are affected by gut fill and are subject to variation depending on water, feed intake and type of feed prior to being mustered, access to water while yarded, amount of faeces and

\footnotetext{
${ }^{1}$ The content of this supplementary information has not been edited. All risk and liability rest with the authors
} 
urine voided while yarded, time off pasture and weight of uterus and its contents (Hughes 1976). A period of starvation for 24 hours before weighing has been suggested as a requirement if precise weight measurements are required (Hughes 1976) but even that rigorous process does not take into account the weight of a gravid uterus. Weights were adjusted to account for time off pasture and average estimates of wool removed during crutching and shearing before conversion to daily weight gain or loss for specific periods.

For purposes of analysis, the weights during the period between start of mating and scanning were collapsed into a categorical variable with values of 1 for increase in weight and 0 for zero increase or loss of weight. Although it suffers from some imprecision due to the factors listed in the previous paragraph, it is arguably more robust for purposes of analysis and it is less complicated by weight of uterus and contents than weights recorded closer to lambing.

\section{Reference:}

Hughes JG. Short-term variation in animal live weight and reduction of its effect on weighing. Animal Breeding Abstracts 44, 111-8, 1976 